Network Working Group

Request for Comments: 1231

\author{
K. McCloghrie \\ Hughes LAN Systems, Inc. \\ R. Fox \\ Synoptics, Inc. \\ E. Decker \\ cisco Systems, Inc. \\ May 1991
}

IEEE 802.5 Token Ring MIB

Status of this Memo

This memo defines a MIB for 805.5 networks for use with the SNMP protocol. This memo is a product of the Transmission Working Group of the Internet Engineering Task Force (IETF). This RFC specifies an IAB standards track protocol for the Internet community, and requests discussion and suggestions for improvements. Please refer to the current edition of the "IAB Official Protocol standards" for the standardization state and status of this protocol. Distribution of this memo is unlimited.

Table of Contents

1. Abstract ............................ 1

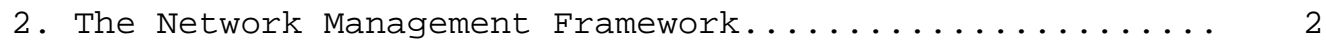

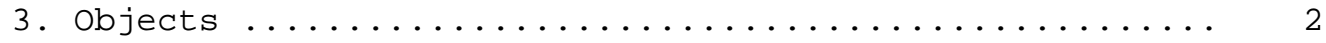

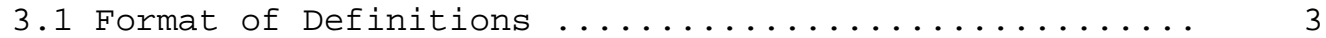

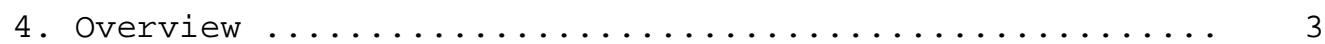

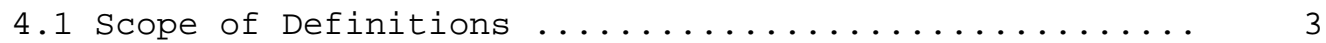

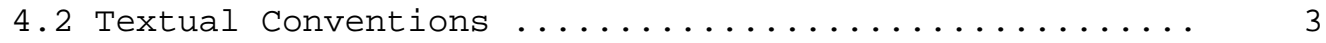

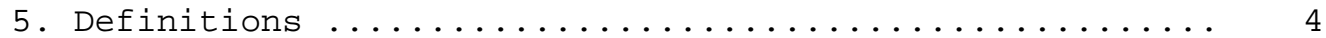

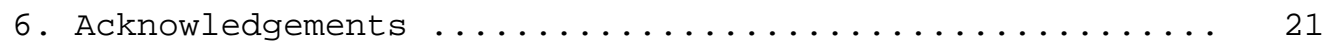

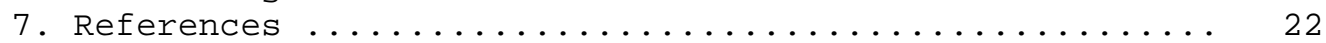

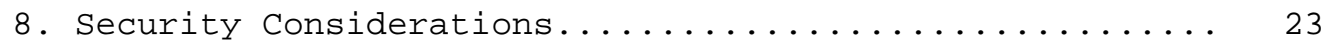

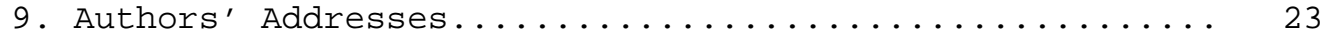

1. Abstract

This memo defines an experimental portion of the Management Information Base (MIB) for use with network management protocols in TCP/IP-based internets. In particular, this memo defines managed objects used for managing subnetworks which use the IEEE 802.5 Token Ring technology described in 802.5 Token Ring Access Method and Physical Layer Specifications, IEEE Standard 802.5-1989.

Transmission Working Group

[Page 1] 
2. The Network Management Framework

The Internet-standard Network Management Framework consists of three components. They are:

RFC 1155 which defines the SMI, the mechanisms used for describing and naming objects for the purpose of management. RFC 1212 defines a more concise description mechanism, which is wholly consistent with the SMI.

RFC 1156 which defines MIB-I, the core set of managed objects for the Internet suite of protocols. RFC 1213, defines MIB-II, an evolution of MIB-I based on implementation experience and new operational requirements.

RFC 1157 which defines the SNMP, the protocol used for network access to managed objects.

The Framework permits new objects to be defined for the purpose of experimentation and evaluation.

3. Objects

Managed objects are accessed via a virtual information store, termed the Management Information Base or MIB. Objects in the MIB are defined using the subset of Abstract Syntax Notation One (ASN.1) [7] defined in the SMI. In particular, each object has a name, a syntax, and an encoding. The name is an object identifier, an administratively assigned name, which specifies an object type. The object type together with an object instance serves to uniquely identify a specific instantiation of the object. For human convenience, we often use a textual string, termed the OBJECT DESCRIPTOR, to also refer to the object type.

The syntax of an object type defines the abstract data structure corresponding to that object type. The ASN.1 language is used for this purpose. However, the SMI [3] purposely restricts the ASN.1 constructs which may be used. These restrictions are explicitly made for simplicity.

The encoding of an object type is simply how that object type is represented using the object type's syntax. Implicitly tied to the notion of an object type's syntax and encoding is how the object type is represented when being transmitted on the network.

The SMI specifies the use of the basic encoding rules of ASN.1 [8], subject to the additional requirements imposed by the SNMP. 


\subsection{Format of Definitions}

Section 5 contains contains the specification of all object types contained in this MIB module. The object types are defined using the conventions defined in the SMI, as amended by the extensions specified in $[9,10]$.

4. Overview

This memo defines three tables: the 802.5 Interface Table, which contains state and parameter information which is specific to 802.5 interfaces, the 802.5 Statistics Table, which contains 802.5 interface statistics, and the 802.5 Timer Table, which contains the values of 802.5-defined timers. A managed system will have one entry in the 802.5 Interface Table and one entry in the 802.5 Statistics Table for each of its 802.5 interfaces. Implementation of the 802.5 Timer Table is optional.

This memo also defines OBJECT IDENTIFIERs, some to identify 802.5 tests, for use with the ifExtnstestTable defined in [11], and some to identify Token Ring interface Chip Sets, for use with the ifExtnsChipset object defined in [11].

\subsection{Scope of Definitions}

All objects defined in this memo are registered in a single subtree within the experimental namespace [3], and are for use with every interface which conforms to the IEEE 802.5 Token Ring Access Method and Physical Layer specifications [10]. At present, this applies to interfaces for which the iftype variable in the Internet-standard MIB $[4,6]$ has the value:

$$
\text { iso88025-tokenRing (9) }
$$

For these interfaces, the value of the ifspecific variable in the MIB-II [6] has the OBJECT IDENTIFIER value:

$$
\text { dot5 OBJECT IDENTIFIER : := \{ experimental } 4\}
$$

as defined below.

\subsection{Textual Conventions}

A new datatype, MacAddress, is introduced as a textual convention in this document. This textual convention has No effect on either the syntax nor the semantics of any managed object. Objects defined using this convention are always encoded by means of the rules that define their primitive type. Hence, no changes to the SMI or the SNMP are 
necessary to accommodate this textual convention which is adopted merely for the convenience of readers.

5. Definitions

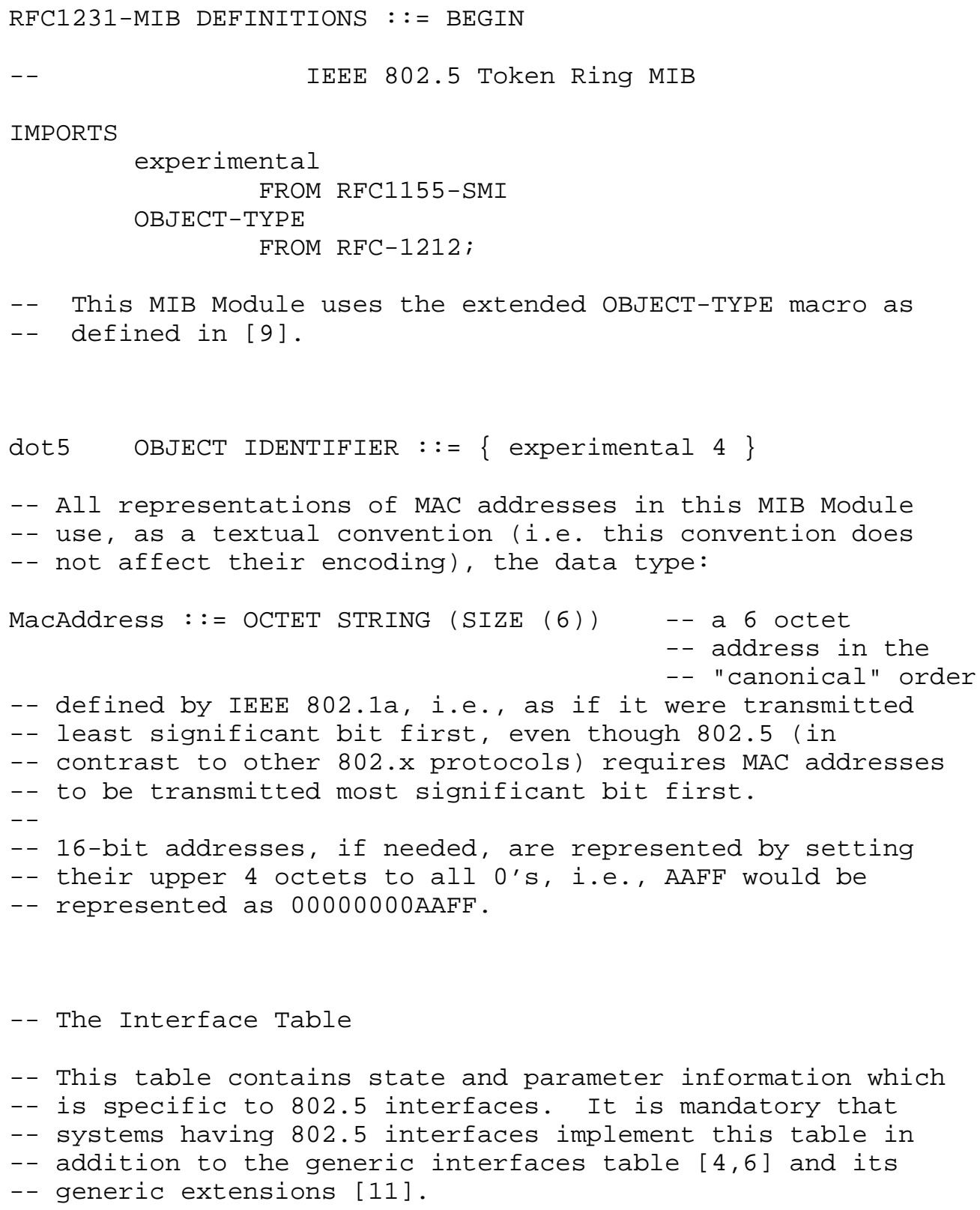




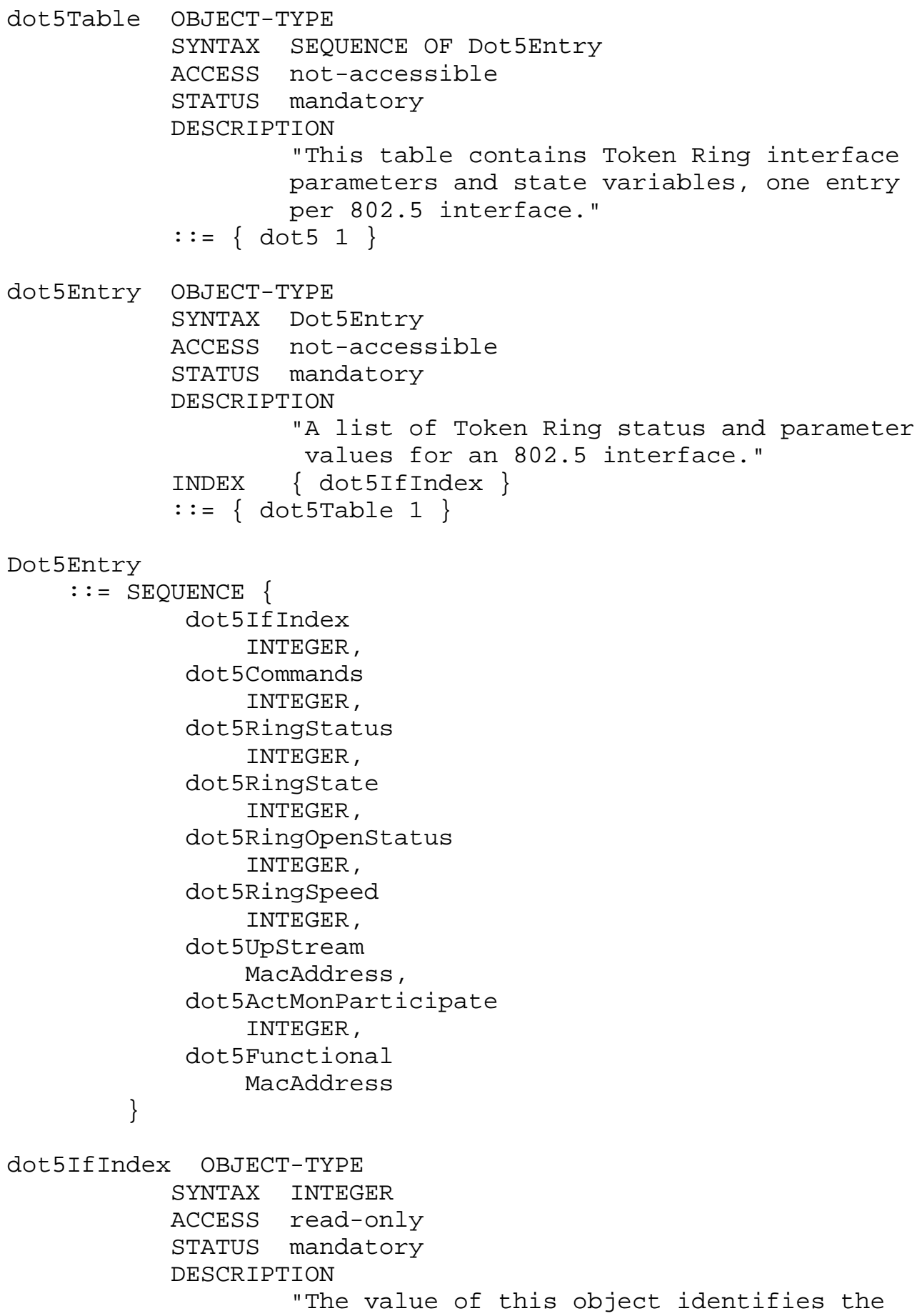


802.5 interface for which this entry contains management information. The value of this object for a particular interface has the same value as the ifIndex object, defined in $[4,6]$, for the same interface."

"The current interface status which can be used to diagnose fluctuating problems that can occur on token rings, after a 


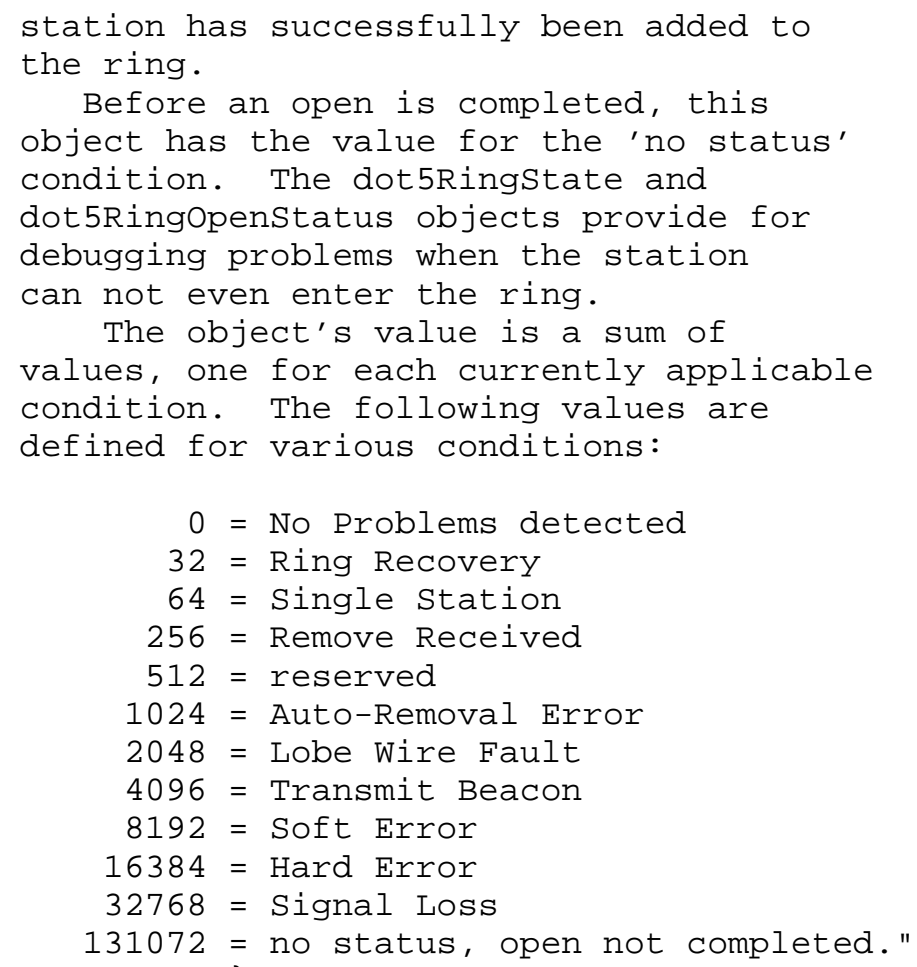




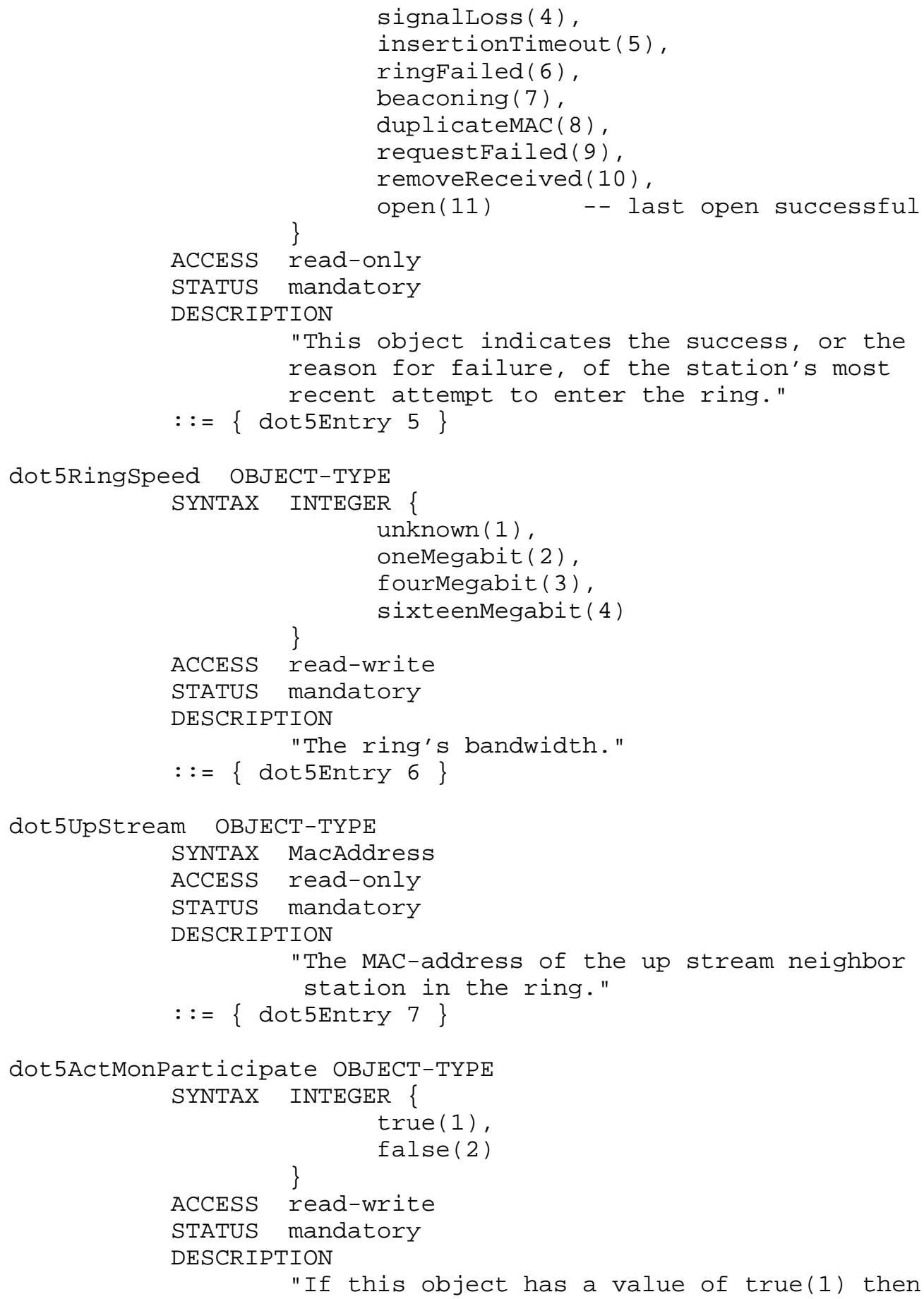




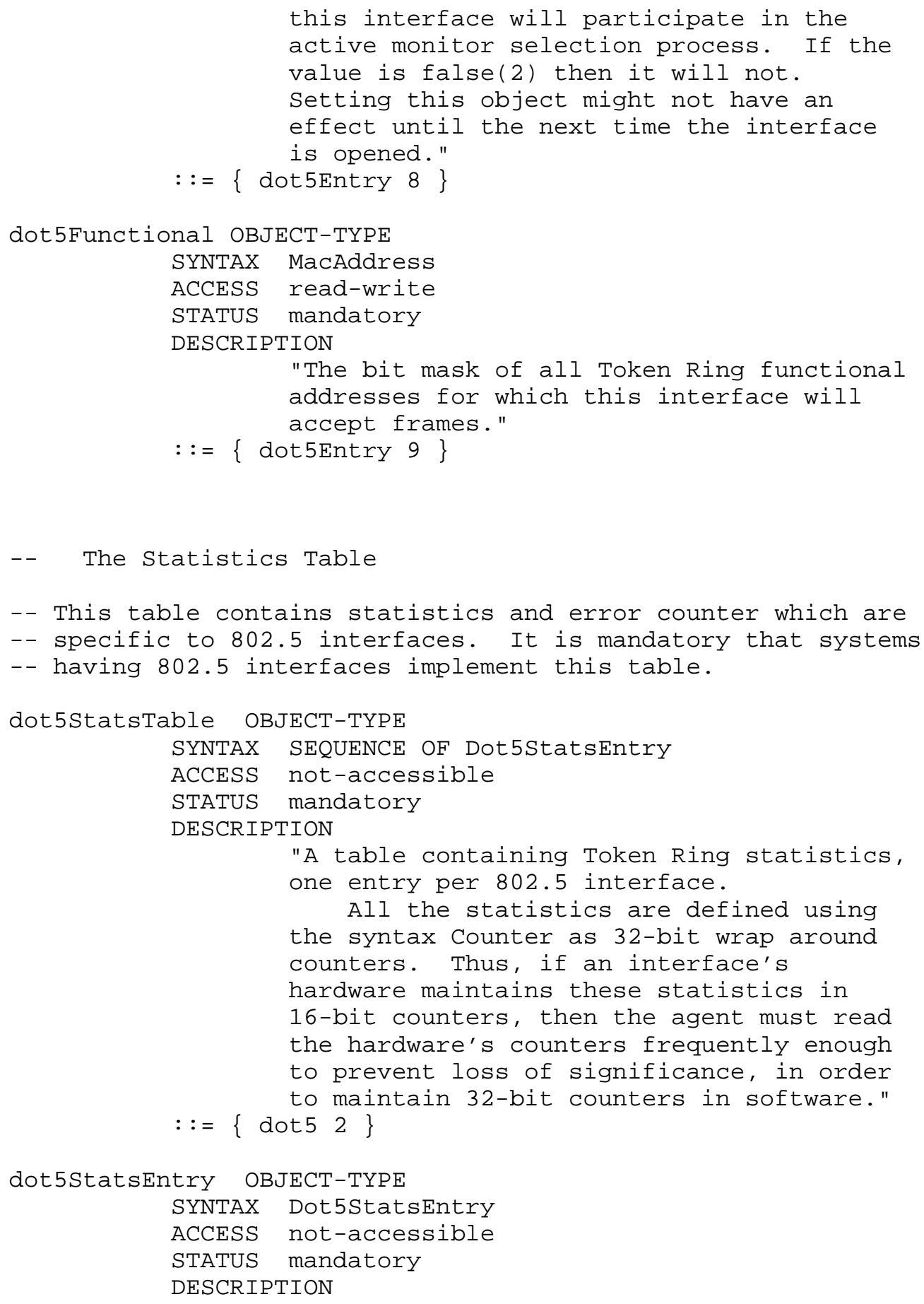




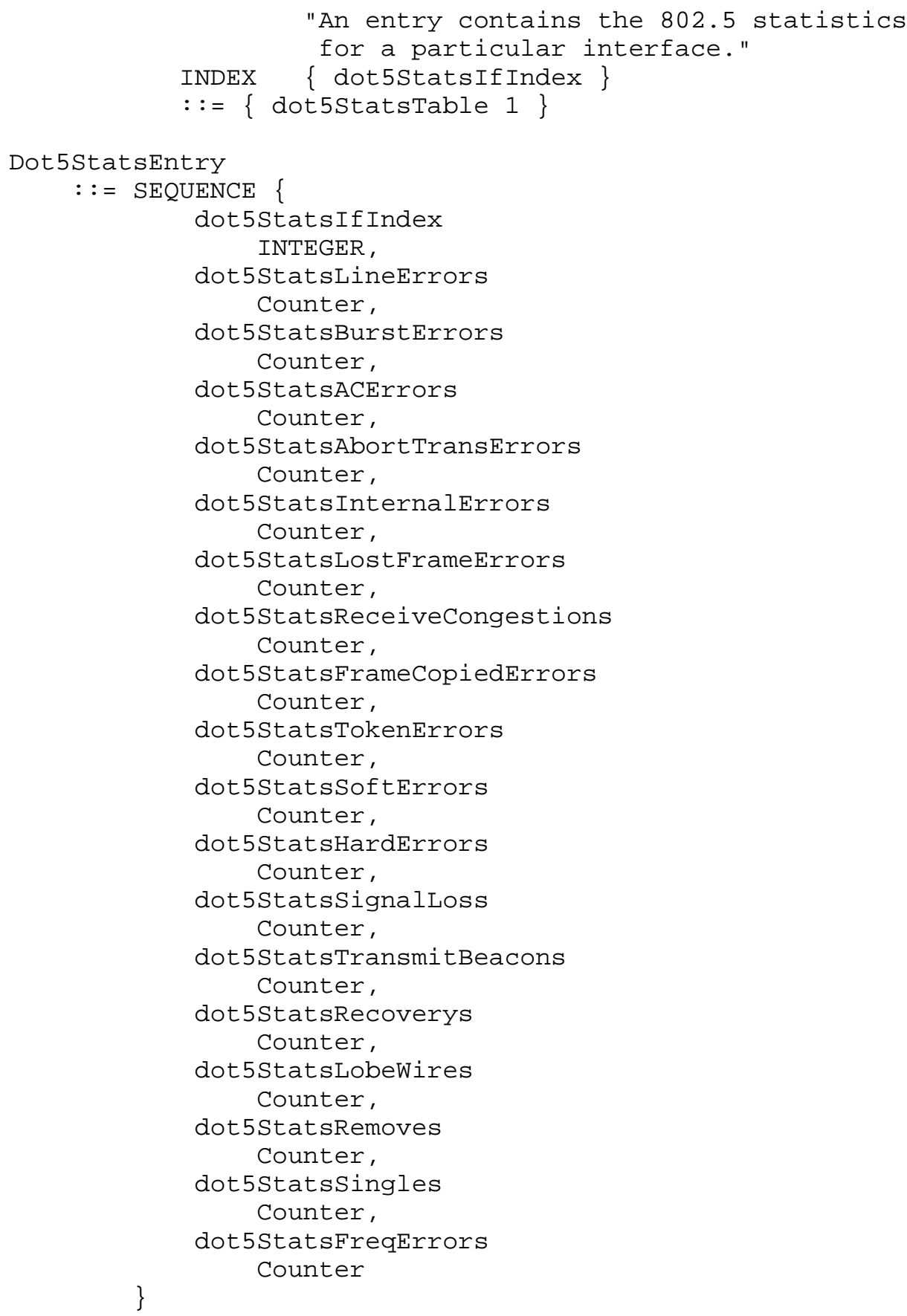




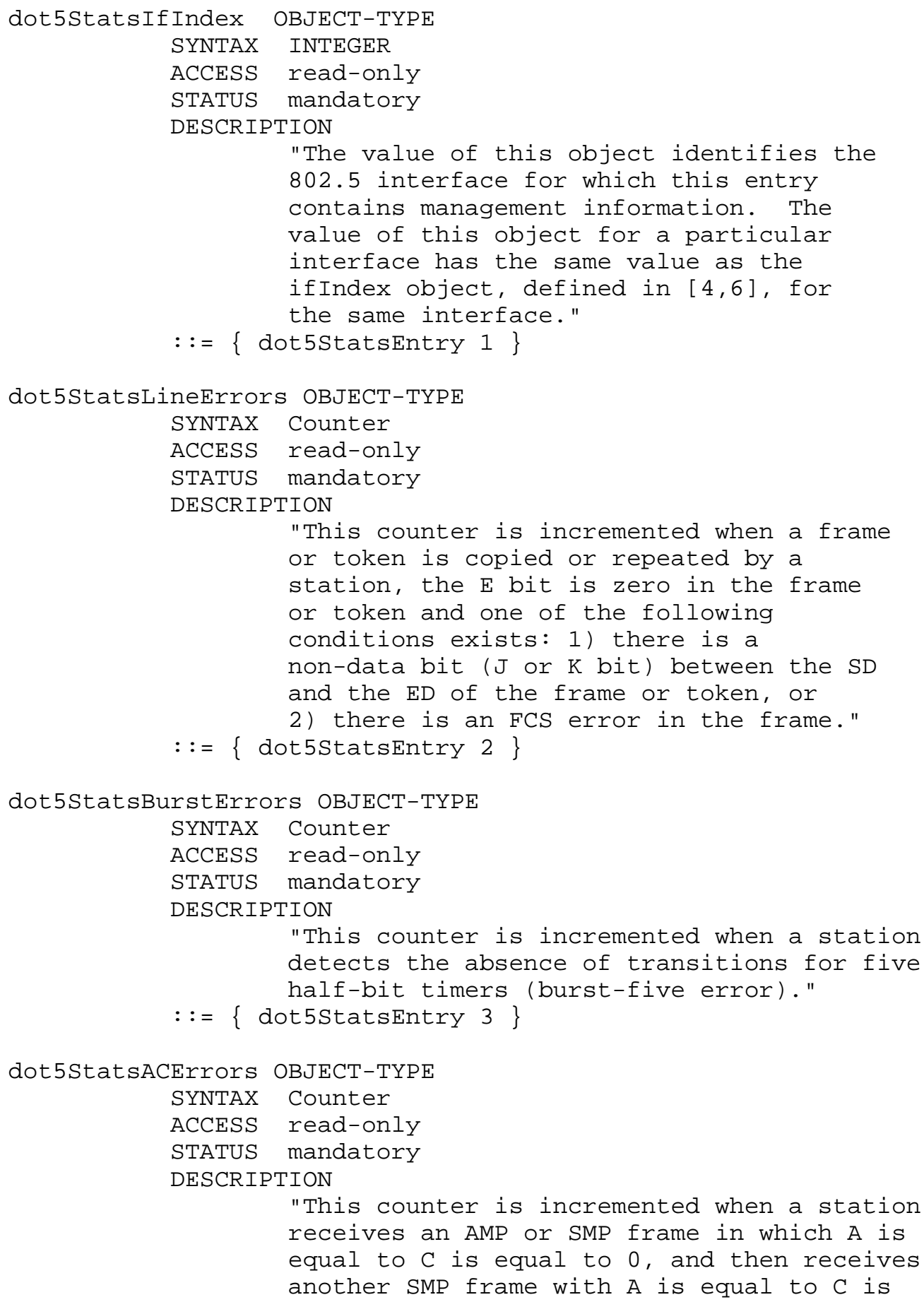




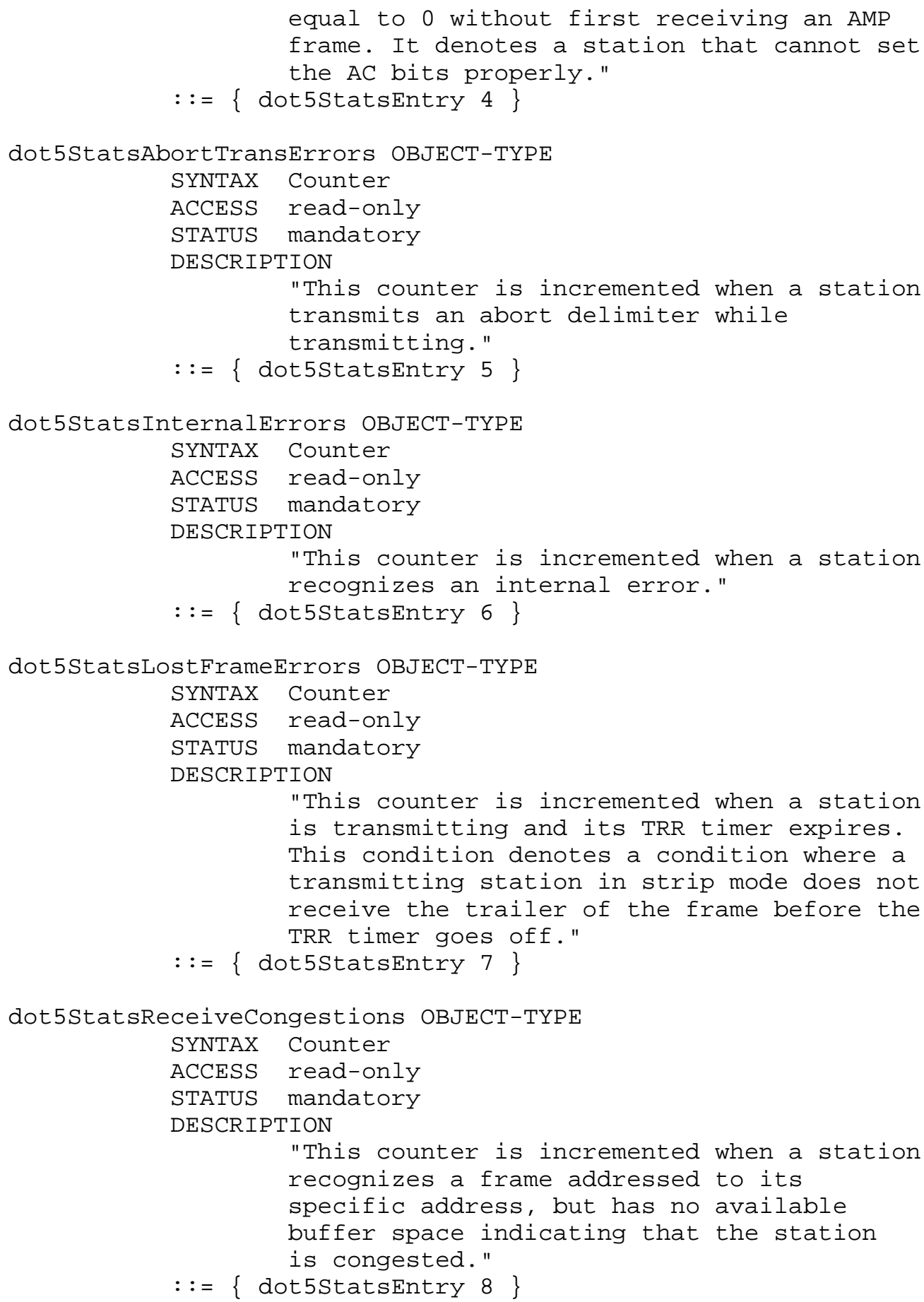

Transmission Working Group 


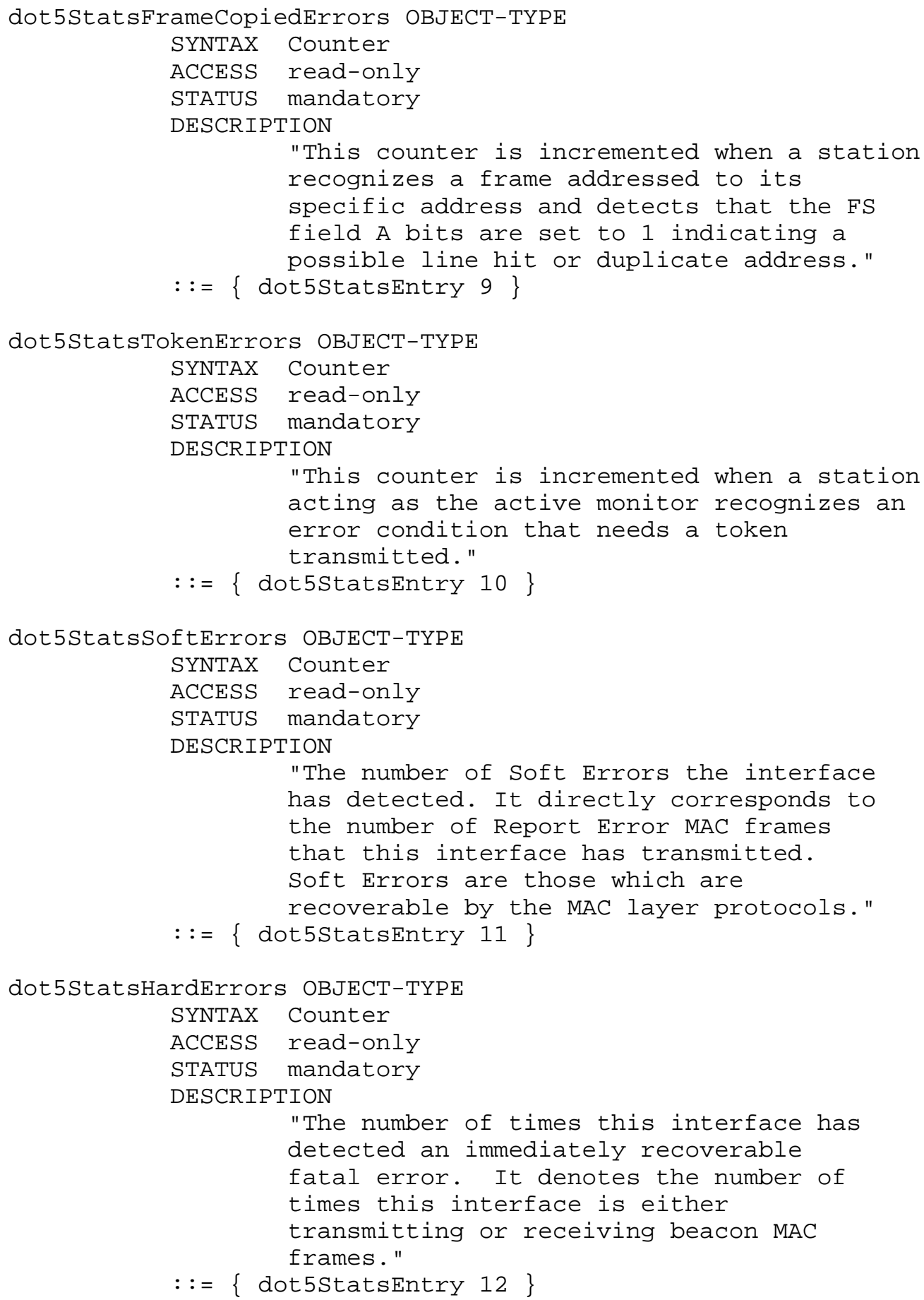

Transmission Working Group 


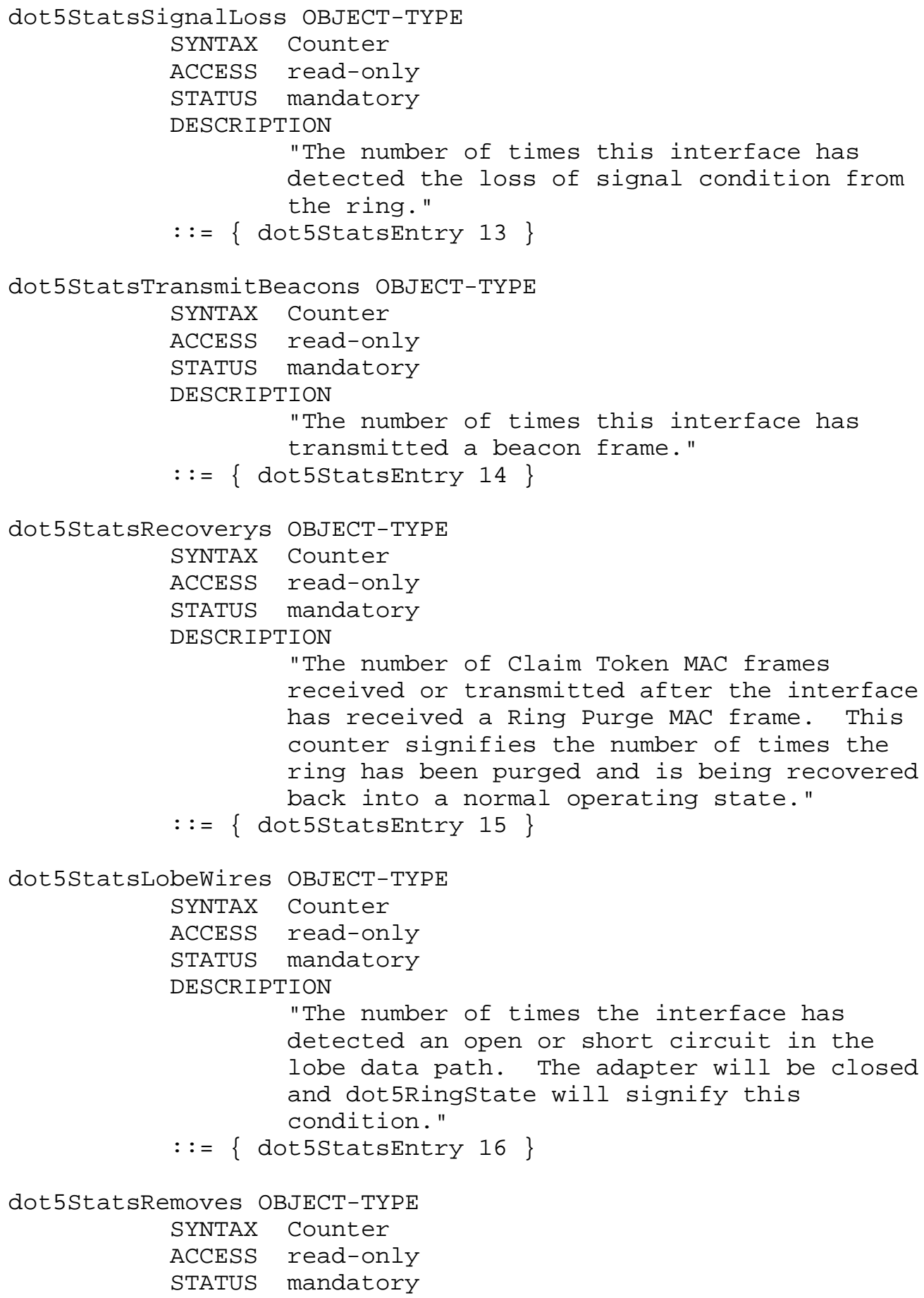




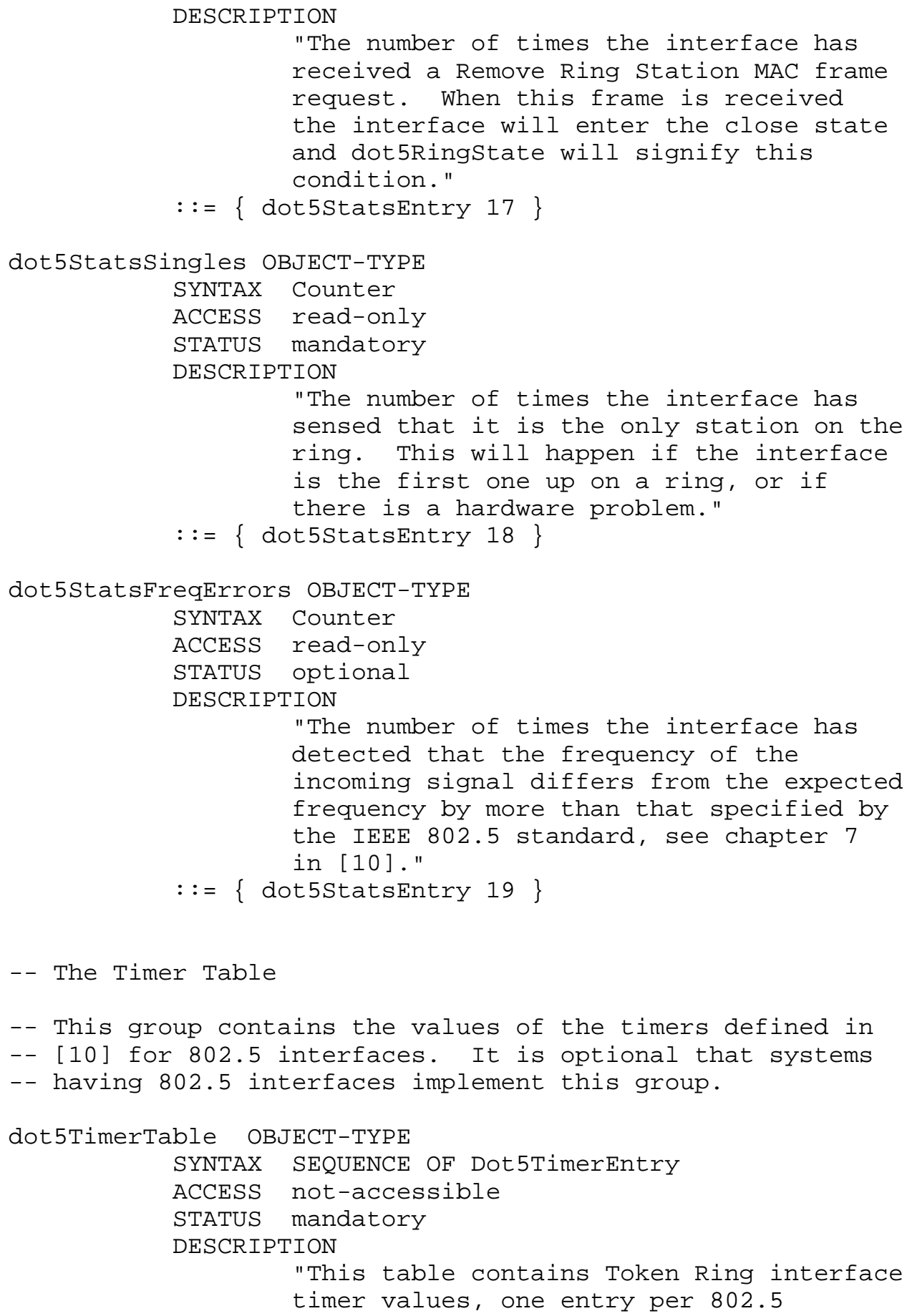




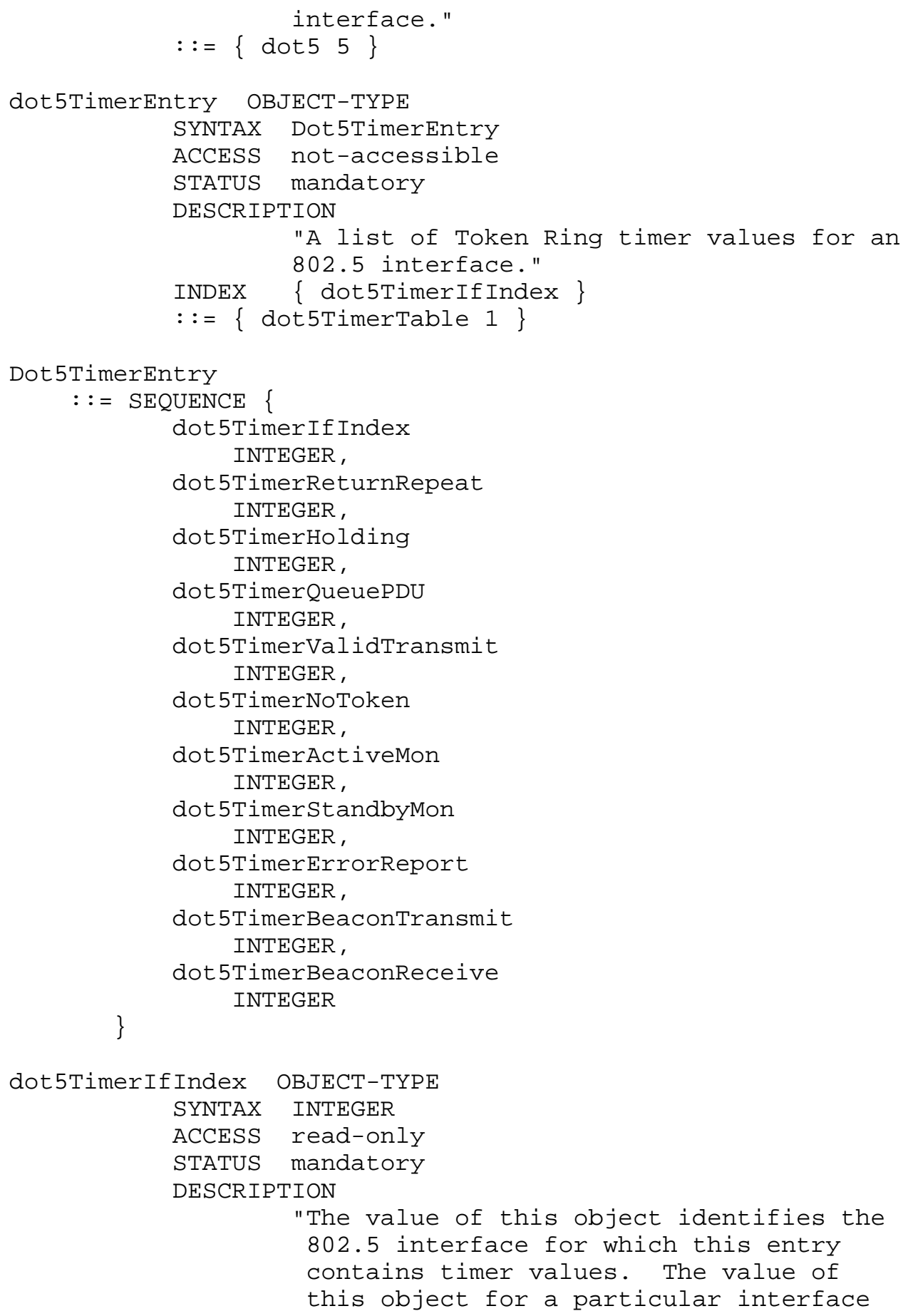




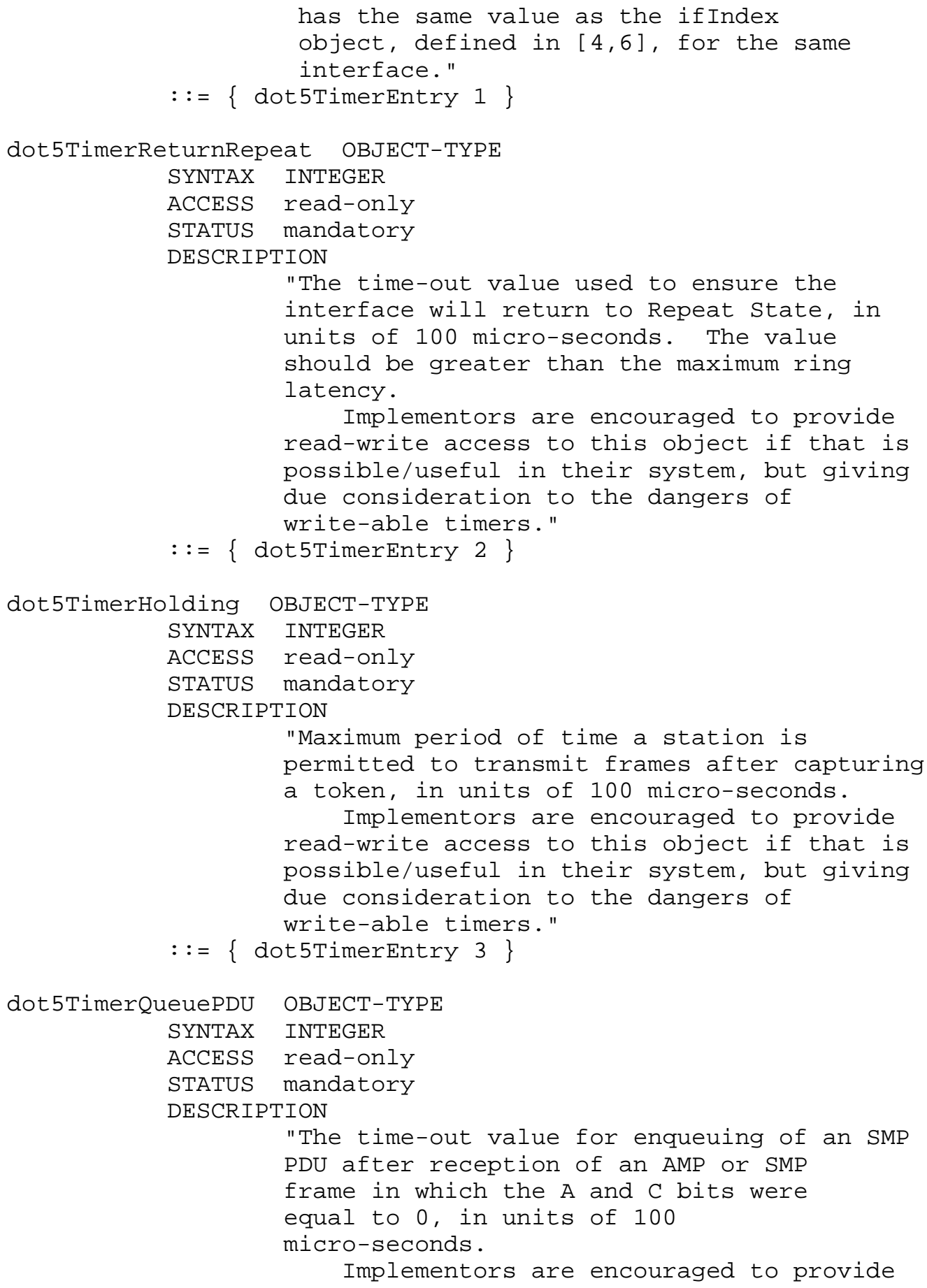




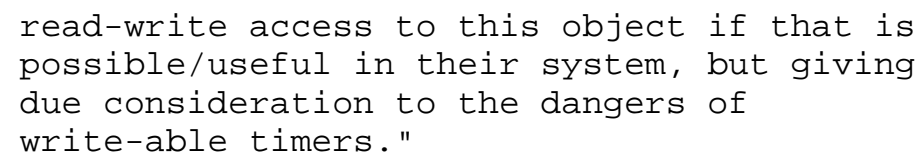




\section{0 micro-seconds.}

Implementors are encouraged to provide read-write access to this object if that is possible/useful in their system, but giving due consideration to the dangers of write-able timers." $::=\{$ dot5TimerEntry 7$\}$

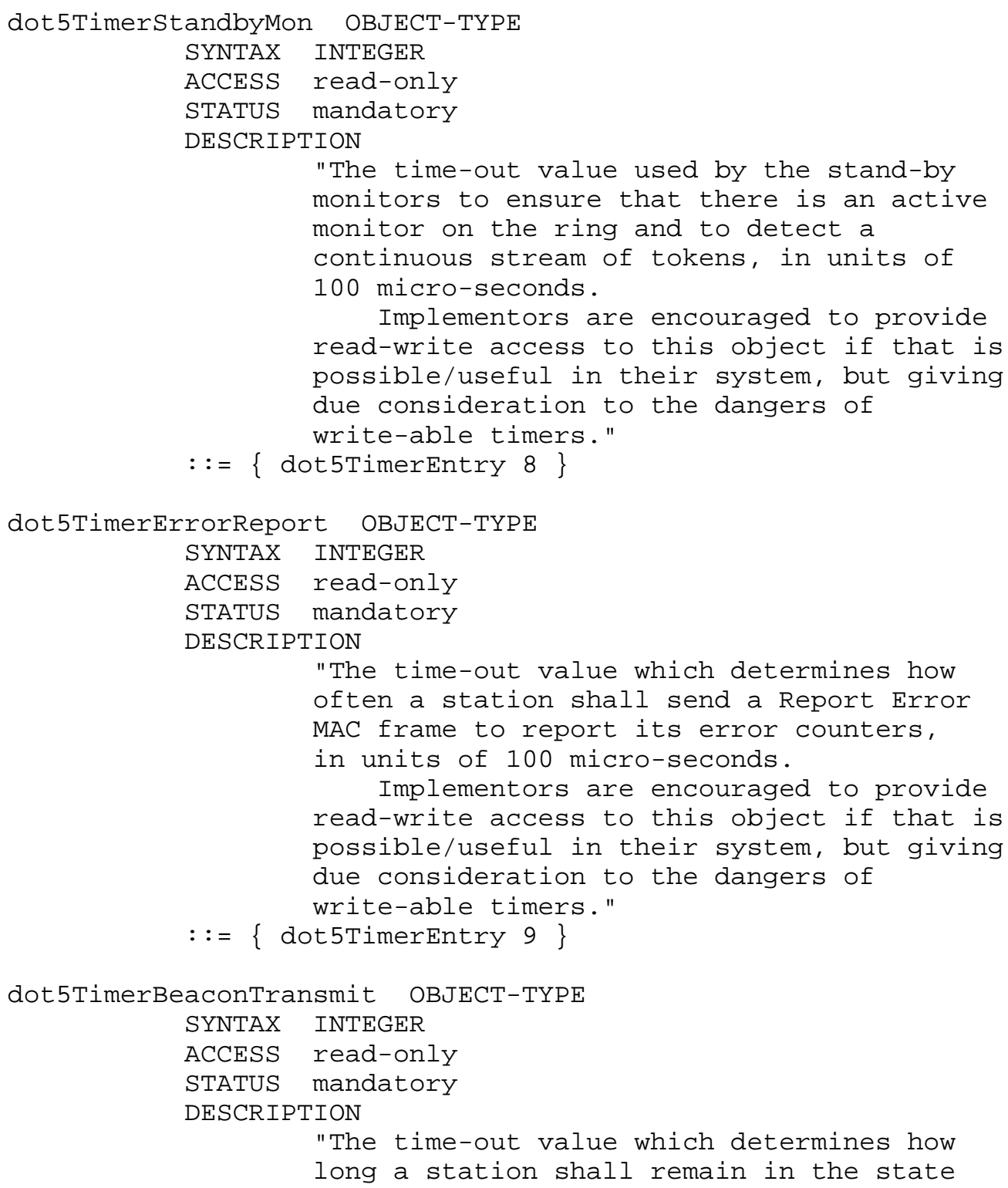




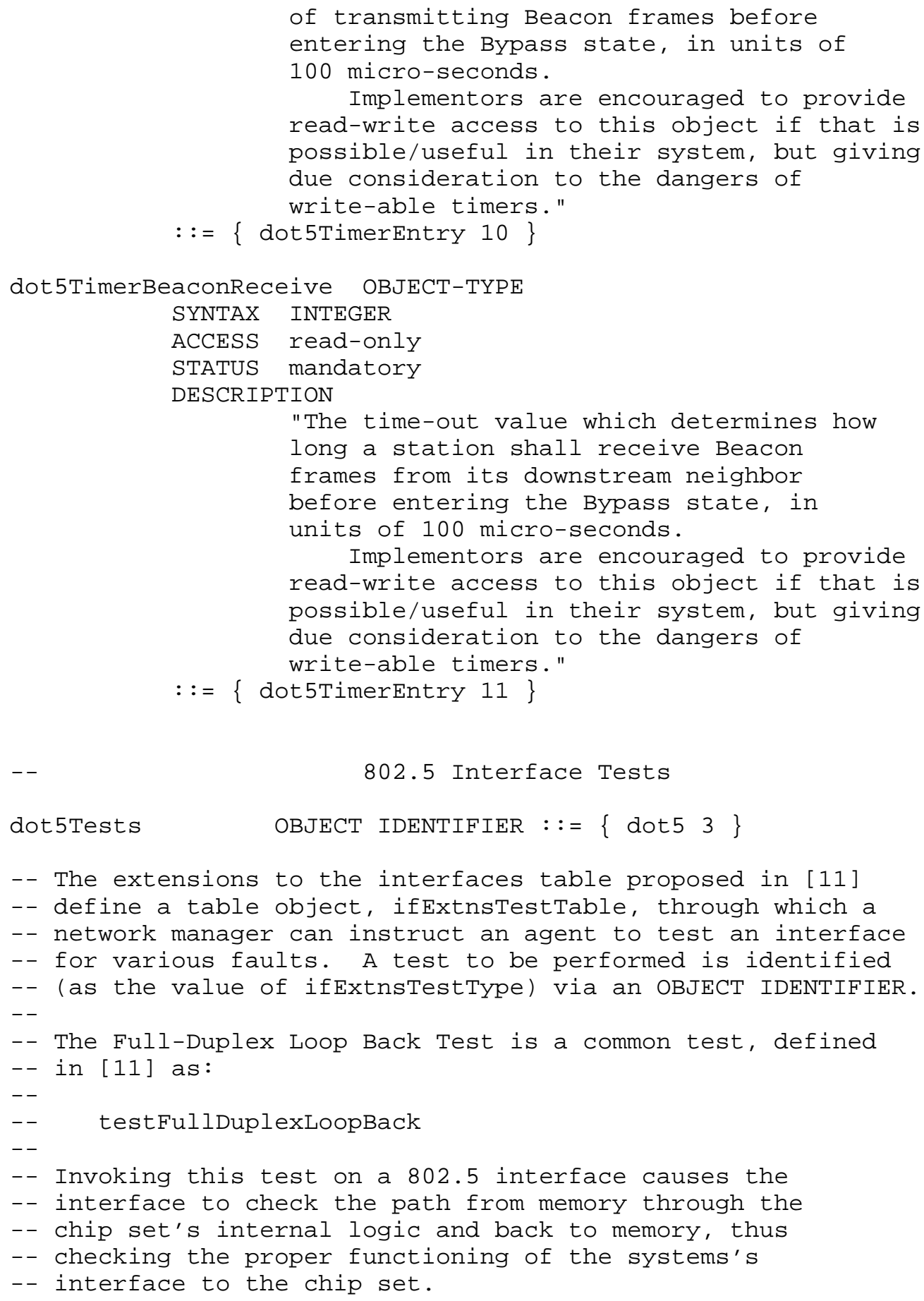


-- The Insert Function test is defined by:

testInsertfunc OBJECT IDENTIFIER : := $\{$ dot5Tests 1$\}$

-- Invoking this test causes the station to test the insert

-- ring logic of the hardware if the station's lobe media

- cable is connected to a wiring concentrator. Note that

-- this command inserts the station into the network, and

-- thus, could cause problems if the station is connected

-- to a operational network.

- $\quad 802.5$ Hardware Chip Sets

dot5chipsets OBJECT IDENTIFIER : := $\{\operatorname{dot} 54\}$

-- The extensions to the interfaces table proposed in [11]

-- define an object, ifExtnschipset, with the syntax of

-- OBJECT IDENTIFIER, to identify the hardware chip set in

-- use by an interface. That definition specifies just

-- one applicable object identifier:

$--$

-- unknownChipset

$--$

-- for use as the value of ifExtnschipset when the specific

-- chip set is unknown.

$--$

-- This MIB defines the following for use as values of

-- ifExtnsChipset:

-- IBM $16 / 4 \mathrm{Mb} / \mathrm{s}$

chipset IBM1 6

OBJECT IDENTIFIER $::=\{\operatorname{dot} 5$ Chipsets 1$\}$

$--\mathrm{TI} 4 \mathrm{Mb} / \mathrm{s}$

chipsetTItms 380

OBJECT IDENTIFIER $::=\{\operatorname{dot} 5$ Chipsets 2$\}$

-- TI $16 / 4 \mathrm{Mb} / \mathrm{s}$

chipsetTItms380c16 OBJECT IDENTIFIER ::= \{dot5Chipsets 3$\}$

END

6. Acknowledgements

This document was produced under the auspices of the IETF's Transmission Working Group. The comments of the following individuals are acknowledged: 
Tom Benkart, Advanced Computer Communications

Stan Froyd, Advanced Computer Communications

Marshall T. Rose, Performance systems International, Inc.

7. References

[1] Cerf, V., "IAB Recommendations for the Development of Internet Network Management Standards", RFC 1052, NRI, April 1988.

[2] Cerf, V., "Report of the Second Ad Hoc Network Management Review Group", RFC 1109, NRI, August 1989.

[3] Rose M., and K. McCloghrie, "Structure and Identification of Management Information for TCP/IP-based internets", RFC 1155, Performance Systems International, Hughes LAN Systems, May 1990.

[4] McCloghrie K., and M. Rose, "Management Information Base for Network Management of TCP/IP-based internets", RFC 1156, Hughes LAN Systems, Performance Systems International, May 1990.

[5] Case, J., Fedor, M., Schoffstall, M., and J. Davin, "Simple Network Management Protocol (SNMP), RFC 1157, SNMP Research, Performance Systems International, Performance systems International, MIT Laboratory for Computer Science, May 1990.

[6] McCloghrie K., and M. Rose, Editors, "Management Information Base for Network Management of TCP/IP-based internets", RFC 1213, Performance Systems International, March 1991.

[7] Information processing systems - Open systems Interconnection Specification of Abstract Syntax Notation One (ASN.1), International Organization for Standardization, International Standard 8824, December 1987.

[8] Information processing systems - Open Systems Interconnection Specification of Basic Encoding Rules for Abstract Notation One (ASN.1), International Organization for Standardization, International Standard 8825, December 1987.

[9] Rose, M., and K. McCloghrie, Editors, "Concise MIB Definitions", RFC 1212, Performance Systems International, Hughes LAN Systems, March 1991.

[10] Token Ring Access Method and Physical Layer Specifications, Institute of Electrical and Electronic Engineers, IEEE Standard $802.5-1989,1989$. 
[11] McCloghrie, K., Editor, "Extensions to the Generic-Interface MIB", RFC 1229, Hughes LAN Systems, May 1991.

8. Security Considerations

Security issues are not discussed in this memo.

9. Authors' Addresses

Keith McCloghrie Hughes LAN Systems, Inc. 1225 Charleston Road Mountain View, CA 94043

Phone: (415) 966-7934

EMail: kzmehls.com

Richard Fox Synoptics, Inc. 4401 Great America Pkwy PO Box 58185

Santa Clara, Cal. 95052

Phone: (408) 764-1372

EMail: rfoxesynoptics.com

Eric Decker

cisco Systems, Inc.

1525 O'Brien Dr.

Menlo Park, CA 94025

Phone: (415) 688-8241

EMail: ciredcisco.com 